\title{
Impact of ABO compatibility on outcomes after heart transplantation in a national cohort during the past decade
}

\author{
Oliver K. Jawitz, AB, ${ }^{a}$ Nicole G. Jawitz, AB, ${ }^{a}$ David D. Yuh, MD, ${ }^{\mathrm{b}}$ and Pramod Bonde, $\mathrm{MD}^{\mathrm{c}}$
}

Background: Immunologic incompatibility has implications for primary graft failure, rejection, and survival in heart transplantation. To our knowledge, this is the first large cohort study investigating the impact of ABO-compatible versus identical blood type matching on post heart transplantation survival.

Methods: We used a nationwide sample (2000-2010) within the United Network for Organ Sharing database. Stratification was between ABO-identical and ABO-compatible heart transplantations for univariate and multivariate analyses. The primary end point was graft failure from all causes. Posttransplant survival was compared between groups using Cox proportional hazard and logistic regression models.

Results: A total of 17,951 patients met inclusion criteria, and 2684 (approximately 15\%) underwent ABOcompatible heart transplantation. ABO-compatible recipients were generally sicker than ABO-identical recipients before transplant because more were status $1 \mathrm{~A}$, in the intensive care unit, and receiving mechanical ventilatory support $(P<.05)$. Univariate analysis correlated ABO-compatible transplants with decreased posttransplant survival and a higher incidence of primary graft failure as cause of death $(P<.05)$. There was no significant difference in acute graft rejection $(P=.53)$. Multivariate analysis, however, did not demonstrate adverse outcomes in terms of decreased graft survival (hazard ratio, $0.99 ; P=.87$ ). Blood type $\mathrm{O}$ donor grafts were associated with poorer outcomes compared with all other types $(P<.05)$.

Conclusions: ABO-compatible transplantation does not result in adverse outcomes with respect to graft survival. Blood type $\mathrm{O}$ donor grafts, however, were associated with decreased survival. This has important implications for current graft allocation policies, particularly for type B recipients. (J Thorac Cardiovasc Surg 2013;146:1239-46)

Cardiac transplantation is an accepted therapy for treating patients with end-stage heart failure. Although transplantation techniques and postoperative management strategies have continued to improve in the past several decades, of the approximately 2000 procedures performed in the United States annually, approximately $10 \%$ of patients do not survive the first year after transplant. ${ }^{1}$ After 1 year, annual death rates approach $4 \%$ and approximately $50 \%$ of heart transplant recipients are alive at 10 years. ${ }^{2}$ There are several risk factors known to be associated with premature death

From the Bonde Artificial Heart and Heart Transplantation Laboratory, ${ }^{a}$ Section of Cardiac Surgery, ${ }^{\mathrm{b}}$ and Yale Center for Advanced Heart Failure, ${ }^{\mathrm{c}}$ Mechanical Circulatory Support and Heart Transplantation, Yale School of Medicine, New Haven, Conn.

Supported, in part, by Health Resources and Services Administration contract 2342005-370011C

Disclosures: Authors have nothing to disclose with regard to commercial support.

Read at the 93rd Annual Meeting of The American Association for Thoracic Surgery, Minneapolis, Minnesota, May 4-8, 2013.

The content is the responsibility of the authors alone and does not necessarily reflect the views or policies of the Department of Health and Human Services, nor does mention of trade names, commercial products, or organizations imply endorsement by the US government.

Received for publication April 26, 2013; revisions received June 22, 2013; accepted for publication June 27, 2013; available ahead of print Aug 26, 2013.

Address for reprints: Pramod Bonde, MD, Surgical Director of Mechanical Circulatory Support, Section of Cardiac Surgery, Yale School of Medicine, 330 Cedar St, 204 Boardman, New Haven, CT 06520 (E-mail: pramod.bonde@yale.edu). $0022-5223 / \$ 36.00$

Copyright (c) 2013 by The American Association for Thoracic Surgery http://dx.doi.org/10.1016/j.jtcvs.2013.06.040 and other complications after cardiac transplantation, including donor cardiac function and preexisting disease, toxicity, systemic infection, ischemic time, and mismatches between donor and recipient heart size, sex, age, and antigenic phenotypes. ${ }^{3}$

Because basic immunologic incompatibility is a clear indication for posttransplant complications, it is common practice to avoid antigenic mismatch when pairing donor hearts with recipients. Human leukocyte antigen (HLA) matching is applied only to highly sensitized individuals listed for heart transplantation, although many centers are using a strategy of "virtual" cross matching. Organ donors and potential recipients are, however, paired based on $\mathrm{ABO}$ blood type matching. There are 3 categories of $\mathrm{ABO}$ matching: $\mathrm{ABO}$ identical, $\mathrm{ABO}$ compatible, and $\mathrm{ABO}$ incompatible. Although adult patients typically do not receive organs from $\mathrm{ABO}$-incompatible donors, avoiding hyperacute graft rejection, recipients sometimes receive hearts from $\mathrm{ABO}$ compatible donors. This is unlike transplant procedures for pediatric recipients, in whom $\mathrm{ABO}$-incompatible grafts are sometimes acceptable because of a delay in the development of natural antibodies to $\mathrm{ABO}$ antigens. ${ }^{4}$

Morbidity and mortality associated with recent increases in donor shortages for all organ transplantation types have led to a renewed interest in ABO-incompatible matching. Although significant progress has been made on this front in the fields of kidney and pediatric heart transplantation, ABO compatibility is largely still a requirement for adult 


\section{Abbreviations and Acronyms \\ $\mathrm{ECMO}=$ extracorporeal membrane oxygenation \\ HLA = human leukocyte antigen \\ ICU = intensive care unit \\ UNOS $=$ United Network for Organ Sharing}

heart transplantation. ${ }^{4}$ Before ABO-incompatible adult heart transplantation can be considered, however, it is important to first solidify our understanding of ABO-identical and ABO-compatible heart transplantation. In the late 1980s and early 1990s, several anecdotal reports suggested unfavorable outcomes among ABO-compatible (nonidentical) adult heart transplants. ${ }^{5,6}$ Since then, however, several small, hospital-based retrospective studies have been conducted, which have largely determined that there are no significant differences in outcomes of $\mathrm{ABO}-$ compatible versus $\mathrm{ABO}$ identical cardiac transplants. ${ }^{6-8}$ The 2012 International Society for Heart and Lung Transplantation Heart Transplant Report listed non-ABO-identical transplants as a borderline significant risk factor for 5-year mortality after transplant. ${ }^{9}$ We believe that it would be clinically useful to compare the medium- with long-term outcomes of ABO-compatible and ABO-identical heart transplants in a large nationwide modern cohort study. Data gleaned from this study could have significant implications for the maximally efficient use of the limited donor pool.

\section{METHODS \\ Data Source}

The United Network for Organ Sharing (UNOS) provided Standard Transplant Analysis and Research files with deidentified donor and recipient transplant data from October 1987 to March 2012 and recipient follow-up data through December 2011. The database includes prospectively collected demographic, donor, operative, and postoperative information for all thoracic transplant recipients in the United States.

\section{Study Design}

We retrospectively reviewed the UNOS database from January 2000 to December 2009. The time points were chosen to identify a modern cohort of heart transplant patients with adequate time for follow-up. All adult ( $\geq 18$ years) single-organ heart transplants were included. Transplants were primarily stratified by transplant donor-recipient $\mathrm{ABO}$ blood type matching (identical vs compatible). Transplants without available data on donor and/or recipient $\mathrm{ABO}$ types were excluded from the study $(\mathrm{n}=1)$.

\section{Outcome Measures}

Demographic and clinical characteristics of all heart transplant donors and recipients were examined. The primary end point was all-cause graft failure during the study period. Secondary outcomes of interest included 30-day mortality, length of hospital stay, graft rejection, and recipient cause of death.

\section{Statistical Analysis}

Baseline demographic and clinical characteristics between the primary study cohorts were compared using the Student $t$ test for continuous variables and the $\chi^{2}$ test for categorical variables. For all Student $t$ tests conducted, normality was assessed using skewness and kurtosis. Survival was modeled using the Kaplan-Meier method, with statistical differences between survival curves assessed using the log-rank (Mantel-Cox) test. Univariate and unadjusted 30-day and 1-, 3-, 5-, and 10-year graft survival analyses were also conducted using the $\chi^{2}$ test. Multivariate analysis was conducted using both the Cox proportional hazards regression model and a logistic regression model. To adjust for potential confounders and accurately determine factors associated with decreased graft survival, variables describing baseline demographic and clinical characteristics that were significantly different $(P<.05)$ between the 2 study cohorts on univariate analysis were included in the multivariate models. For the logistic regression analysis, variables were removed from the model in a stepwise manner until all included variables (except $\mathrm{ABO}$ compatibility and the variable of interest) were statistically significant $(P<.05)$.

Statistical significance was established at $P<.05$ ( 2 tailed), and all hazard ratios are presented with $95 \%$ confidence intervals. All statistical analysis was generated using SAS software, version 9.3, of the SAS System for Windows (SAS Institute Inc, Cary, NC).

\section{RESULTS}

The UNOS database contained records of 15,267 ABO-identical transplants and 2684 ABO-compatible transplants during the study period from January 2000 to December 2009 that fit the study's inclusion criteria (Table 1). Of the transplant recipients with blood types A, $\mathrm{B}$, and $\mathrm{AB}$, the frequency of $\mathrm{ABO}$-compatible transplants was $17.0 \%, 32.8 \%$, and $61.8 \%$, respectively. Blood type $\mathrm{O}$ recipients can only receive $\mathrm{ABO}$-identical grafts.

The baseline demographic characteristics of both donors and recipients from these transplant surgical procedures are summarized in Tables 2 and 3, respectively. The allograft donors from both cohorts were well matched based on sex, age, mean left ventricular ejection fraction, cause of death, and a history of hypertension, diabetes, and cigarette use. There was a significant difference $(P<.05)$ between the 2 groups in terms of donor ethnicity and history of cancer.

The baseline demographic and clinical characteristics of heart recipients in the ABO-identical and ABO-compatible cohorts differed $(P<.05)$ with respect to sex, age, ethnicity, wait list status at transplant, status before transplant (in intensive care unit [ICU], in hospital, or not hospitalized), life support before transplant, and mean graft ischemic time and total bilirubin. More ABO-compatible transplant recipients were wait list status $1 \mathrm{~A}(50.3 \%)$ than ABO-identical transplant recipients $(28.3 \%, P<.001)$.

TABLE 1. ABO blood group distribution

\begin{tabular}{|c|c|c|c|c|c|c|}
\hline & \multicolumn{6}{|c|}{ Donor blood type } \\
\hline & $\mathbf{A}$ & $\mathbf{A B}$ & B & $\mathbf{O}$ & $\begin{array}{c}\text { Total } \\
\text { identical* }\end{array}$ & $\begin{array}{c}\text { Total } \\
\text { compatible* }\end{array}$ \\
\hline \multicolumn{7}{|c|}{ Recipient blood type } \\
\hline A & 6340 & 0 & 0 & 1302 & $6340(83.0)$ & $1302(17.0)$ \\
\hline $\mathrm{AB}$ & 274 & 342 & 189 & 90 & $342(38.2)$ & $553(61.8)$ \\
\hline B & 0 & 0 & 1696 & 829 & $1696(67.2)$ & $829(32.8)$ \\
\hline $\mathrm{O}$ & 0 & 0 & 0 & 6889 & $6889(100)$ & $0(0)$ \\
\hline
\end{tabular}

*Total identical and total compatible measured as proportion of each recipient blood type. 
TABLE 2. Donor characteristics stratified by ABO blood type matching

\begin{tabular}{|c|c|c|c|}
\hline Variable & $\begin{array}{l}\text { ABO identical } \\
(\mathbf{n}=15,267)^{*}\end{array}$ & $\begin{array}{c}\text { ABO } \\
\text { compatible } \\
(\mathbf{n}=\mathbf{2 6 8 4})^{*}\end{array}$ & $\begin{array}{c}P \\
\text { value } \dagger\end{array}$ \\
\hline Female sex & $4306(28.2)$ & 787 (29.3) & .24 \\
\hline Mean (SD) donor age, y & $31.54 \pm 12.35$ & $31.40 \pm 12.53$ & .58 \\
\hline \multicolumn{4}{|l|}{ Ethnicity } \\
\hline White & $10,687(70.0)$ & $1697(63.3)$ & $<.001$ \\
\hline Black & $1938(12.7)$ & $387(14.4)$ & .01 \\
\hline Hispanic or Latino & $2259(14.8)$ & $518(19.3)$ & $<.001$ \\
\hline Asian & $213(1.4)$ & $46(1.7)$ & .20 \\
\hline History of hypertension & $1829(12.0)$ & $336(12.6)$ & .42 \\
\hline History of cancer & $254(1.7)$ & $64(2.4)$ & .01 \\
\hline History of diabetes & $343(2.3)$ & $68(2.5)$ & .35 \\
\hline History of cigarette use & $3903(25.8)$ & $698(26.2)$ & .67 \\
\hline \multicolumn{4}{|l|}{ Cause of death } \\
\hline Anoxia & $1562(10.2)$ & $288(10.7)$ & .44 \\
\hline Cerebrovascular/stroke & $3839(25.2)$ & $713(26.6)$ & .12 \\
\hline Head trauma & $9427(61.8)$ & $1615(60.2)$ & .12 \\
\hline CNS tumor & $158(1.0)$ & $25(0.93)$ & .62 \\
\hline Mean (SD) LVEF & $61.57 \pm 7.83$ & $61.62 \pm 8.02$ & .74 \\
\hline
\end{tabular}

In addition, $40.4 \%$ of ABO-compatible recipients were in the ICU before transplant compared with only $28.3 \%$ of ABO-identical recipients $(P<.05)$. When compared with ABO-identical transplant recipients, ABO-compatible transplant recipients were more frequently on life support before transplant $(P<.05)$, including extracorporeal membrane oxygenation (ECMO), intra-aortic balloon pump, intravenous inotropes, and ventilator support. There was no statistical difference between the 2 groups in terms of ventricular assist device use $(P=.266)$. Graft ischemic time and total bilirubin also differed between the 2 study cohorts $(P<.001)$; ABO-identical transplant recipients had a longer mean ischemic time and lower total bilirubin ( 3.23 hours, $1.25 \mathrm{mg} / \mathrm{dL}$ ) compared with ABO-compatible transplant recipients (3.11 hours, 1.48 $\mathrm{mg} / \mathrm{dL}$ ).

Table 4 shows unadjusted 30-day and 1-, 3-, 5-, and 10-year graft survival for $\mathrm{ABO}$-identical and $\mathrm{ABO}$-compatible heart transplant recipients. Recipients of $\mathrm{ABO}$-identical grafts had increased graft survival $(P<.05)$ compared with ABOcompatible recipients at 30 days $(94.4 \%$ vs $93.3 \%)$, 1 year $(87.0 \%$ vs $84.4 \%)$ ), 3 years $(76.3 \%$ vs $73.4 \%)$, and 5 years $(63.1 \%$ vs $60.0 \%)$ after transplant. There was no statistically significant difference in graft survival at 10 years after transplant $(P=.21)$. In addition, there was no difference in the incidence of rejection between transplant and discharge $(P=.53)$ and mean length of stay as well as length of stay between transplant and discharge $(P=.97)$.
TABLE 3. Recipient characteristics stratified by ABO blood type matching

\begin{tabular}{|c|c|c|c|}
\hline Variable & $\begin{array}{l}\text { ABO identical } \\
(N=15,267)^{*}\end{array}$ & $\begin{array}{l}\text { ABO compatible } \\
(\mathbf{N}=\mathbf{2 6 8 4})^{*}\end{array}$ & $\begin{array}{c}P \\
\text { value }\end{array}$ \\
\hline Female sex & $3584(23.5)$ & $708(26.4)$ & .001 \\
\hline Mean (SD) recipient age, y & $51.91 \pm 12.26$ & $51.12 \pm 12.87$ & $<.001$ \\
\hline \multicolumn{4}{|l|}{ Ethnicity } \\
\hline White & $11,286(73.9)$ & $1882(70.1)$ & $<.001$ \\
\hline Black & $2399(15.7)$ & $498(18.6)$ & $<.001$ \\
\hline Hispanic or Latino & $1110(7.3)$ & $179(6.7)$ & .27 \\
\hline Asian & $311(2.0)$ & $102(3.8)$ & $<.001$ \\
\hline \multicolumn{4}{|l|}{ Wait list status at transplant } \\
\hline $1 \mathrm{~A}$ & $5771(37.8)$ & $1350(50.3)$ & $<.001$ \\
\hline 1B & $6040(39.6)$ & $928(34.6)$ & $<.001$ \\
\hline 2 & $3450(22.6)$ & $405(15.1)$ & $<.001$ \\
\hline \multicolumn{4}{|l|}{ Status before transplant } \\
\hline In ICU & $4321(28.3)$ & $1083(40.4)$ & $<.001$ \\
\hline In hospital (not ICU) & $2853(18.7)$ & $545(20.3)$ & .05 \\
\hline Not in hospital & $8093(53.0)$ & $1056(39.3)$ & $<.001$ \\
\hline \multicolumn{4}{|l|}{ Life support at transplant } \\
\hline ECMO & $67(0.44)$ & $25(0.93)$ & .001 \\
\hline IABP & $737(4.8)$ & $234(8.7)$ & $<.001$ \\
\hline IV inotropes & $6,786(44.5)$ & $1,330(49.6)$ & $<.001$ \\
\hline Inhaled NO & $36(0.24)$ & $7(0.26)$ & .81 \\
\hline Ventilatory support & $387(2.5)$ & $122(4.5)$ & $<.001$ \\
\hline VAD & 3609 (23.6) & $608(22.7)$ & .27 \\
\hline History of dialysis & $368(2.4)$ & $76(2.8)$ & .2 \\
\hline History of cardiac surgery & $2918(19.1)$ & $543(20.2)$ & .18 \\
\hline History of malignancy & $739(4.8)$ & $137(5.1)$ & .56 \\
\hline History of diabetes & $3460(22.7)$ & $600(22.4)$ & .73 \\
\hline History of cigarette use & $3848(25.2)$ & $700(26.1)$ & .34 \\
\hline Mean (SD) ischemic time, $\mathrm{h}$ & $3.23 \pm 1.05$ & $3.11 \pm 1.00$ & $<.001$ \\
\hline $\begin{array}{l}\text { Mean }(\mathrm{SD}) \text { serum creatinine } \\
\text { at } \mathrm{Tx}, \mathrm{mg} / \mathrm{dL}\end{array}$ & $1.31 \pm 0.56$ & $1.33 \pm 0.63$ & .14 \\
\hline $\begin{array}{l}\text { Mean (SD) total bilirubin, } \\
\mathrm{mg} / \mathrm{dL}\end{array}$ & $1.23 \pm 1.94$ & $1.37 \pm 2.01$ & $<.001$ \\
\hline CMV IgG positive & $8675(62.6)$ & $1567(63.7)$ & .32 \\
\hline CMV IgM positive & $816(8.8)$ & $164(9.6)$ & .24 \\
\hline
\end{tabular}

$I C U$, Intensive care unit; $E C M O$, extracorporeal membrane oxygenation; $I A B P$, intraaortic balloon pump; $I V$, intravenous; $N O$, nitric oxide; $V A D$, ventricular assist device; $T x$, treatment; $C M V$, cytomegalovirus; $S D$, standard deviation; $\operatorname{Ig} G$, immunoglobulin G; IgM, immunoglobulin M. *Some patients were excluded from each analysis because of missing data fields or erroneously imputed data in the database. $\dagger P$ value based on Student $t$ test for continuous variables and the $\chi^{2}$ test for categorical variables $(P<.05$ is considered statistically significant $)$.

Transplant recipient cause of death was similar between ABO-identical and ABO-compatible recipients, except for mortality due to primary graft failure and malignancy (Table 5). More ABO-compatible heart recipients died from primary graft failure than $\mathrm{ABO}$-identical recipients $(8.7 \%$ vs $5.8 \% ; P=.003)$. Interestingly, ABO-identical transplant recipients showed a greater incidence of death due to malignancy than the ABO-compatible cohort $(9.8 \%$ vs $6.6 \% ; P=.007)$.

When graft survival was compared between the 2 study cohorts using the Kaplan-Meier method (Figure 1), ABOidentical recipients showed a slightly higher degree of graft 
TABLE 4. Outcomes stratified by ABO blood type matching

\begin{tabular}{lccc}
\hline \multicolumn{1}{c}{ Variable } & $\begin{array}{c}\text { ABO identical } \\
(\mathbf{N}=\mathbf{1 5 , 2 6 7 )}\end{array}$ & $\begin{array}{c}\text { ABO compatible } \\
(\mathbf{N}=\mathbf{2 6 8 4})\end{array}$ & $\begin{array}{c}\boldsymbol{P} \\
\text { value* }^{*}\end{array}$ \\
\hline 30-d Survival & $14,396(94.4)$ & $2500(93.3)$ & .02 \\
1-y Survival & $13,241(87.0)$ & $2258(84.4)$ & $<.001$ \\
3-y Survival & $10,199(76.3)$ & $1720(73.4)$ & .003 \\
5-y Survival & $6960(63.1)$ & $1140(59.9)$ & .009 \\
10-y Survival & $1147(17.8)$ & $187(16.3)$ & .21 \\
$\begin{array}{l}\text { Rejection between transplant } \\
\quad\end{array}$ & $1319(8.6)$ & $222(8.3)$ & .53 \\
$\quad$ & & \\
$\begin{array}{l}\text { Meand discharge }(S D) \text { length of stay, } \\
\text { transplant to discharge, d }\end{array}$ & $20.04 \pm 25.86$ & $20.05 \pm 22.14$ & .97 \\
\hline $\begin{array}{l}\text { Survival data based on graft survival time, after transplant; } S D, \text { standard deviation. } \\
* P \text { value based on Student } t \text { test for continuous variables and the } \chi^{2} \text { test for categorical }\end{array}$ &
\end{tabular}

survival, although the log-rank test showed that this difference was not statistically significant $(P=.09)$.

The multivariate Cox proportional hazards regression model (Table 6) demonstrated 6 variables of significance $(P<.05)$ for the outcome measure of graft failure: recipient ethnicity, ventilatory support at transplant, pretransplant ECMO use, graft ischemic time, total bilirubin, and patient status before transplant (in ICU, in hospital, or not hospitalized). Although univariate analysis showed ABO blood type matching (identical vs compatible) to have a significant impact on the incidence of graft failure, this effect was eliminated when controlling for potential confounders in the multivariate model (hazard ratio [ABO compatible], $0.991 ; P=.865)$.

In the multivariate logistic regression model showing risk factors for 30-day graft failure posttransplant, variables of significance $(P<.05)$ were life support at transplant, including intravenous inotropes, ventilator support, and pretransplant ECMO use; ischemic time; wait list status at transplant; status before transplant (in ICU, in hospital, or not hospitalized); and total bilirubin (Table 7). Once again,

TABLE 5. Recipient cause of death stratified by ABO blood type matching

\begin{tabular}{lccc}
\hline \multicolumn{1}{c}{ Variable } & $\begin{array}{c}\text { ABO identical } \\
(\mathbf{N}=\mathbf{4 0 0 0})\end{array}$ & $\begin{array}{c}\text { ABO compatible } \\
(\mathbf{N}=\mathbf{7 2 4})\end{array}$ & $\boldsymbol{P}$ value* \\
\hline Graft failure: all causes & $709(17.7)$ & $149(20.6)$ & .07 \\
$\quad$ Primary failure & $231(5.8)$ & $63(8.7)$ & .003 \\
Acute rejection & $252(6.3)$ & $41(5.7)$ & .51 \\
Chronic rejection & $108(2.7)$ & $25(3.5)$ & .26 \\
Infection & $615(15.4)$ & $119(16.4)$ & .47 \\
Cardiovascular & $773(19.3)$ & $144(19.9)$ & .72 \\
Pulmonary & $259(6.5)$ & $45(6.2)$ & .79 \\
Cerebrovascular & $180(4.5)$ & $23(3.2)$ & .11 \\
Hemorrhage & $98(2.5)$ & $17(2.4)$ & .87 \\
Malignancy & $392(9.8)$ & $48(6.6)$ & .007 \\
Renal failure & $102(2.6)$ & $23(3.2)$ & .33 \\
Multiple-organ failure & $417(10.4)$ & $78(10.8)$ & .78 \\
\hline
\end{tabular}

$* P$ value based on the $\chi^{2}$ test for categorical variables $(P<.05$ is considered statistically significant).

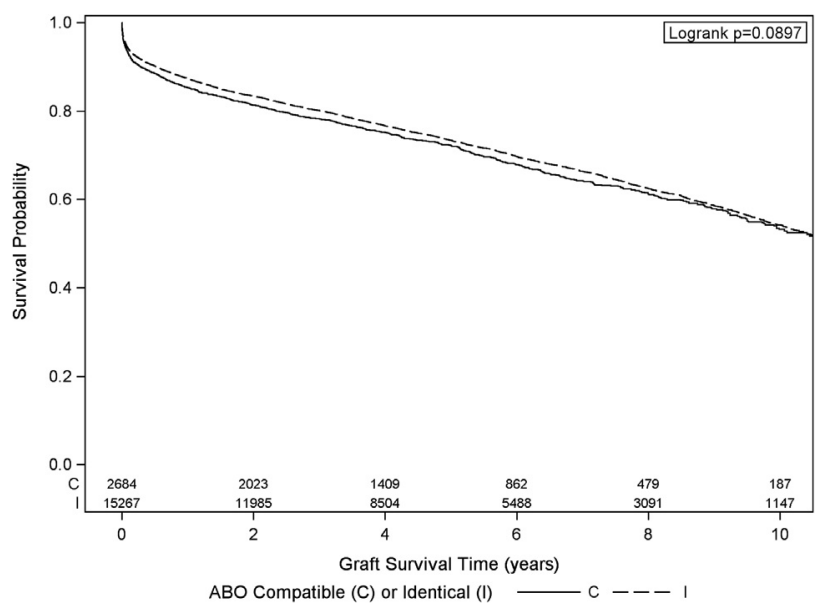

FIGURE 1. Kaplan-Meier graft survival analysis, ABO-compatible $(C)$ versus ABO-identical (I) transplants. Solid line, ABO-compatible transplants; dashed line, $\mathrm{ABO}$-identical transplants. A table is given with the number of patients at risk at each time point. The $P$ value corresponds to Mantel-Cox log-rank test results.

when controlling for potential confounding variables, $\mathrm{ABO}$ matching (identical vs compatible) was not statistically significant $(P=.08)$.

Posttransplant graft survival was also compared among different donor $\mathrm{ABO}$ blood groups using the KaplanMeier method (Figure 2). This analysis demonstrated decreased graft survival associated with type $\mathrm{O}$ donors and increased survival associated with type A donors $(P<.05)$ when compared with all other blood types. Type $\mathrm{B}$ and $\mathrm{AB}$ donors were not associated with either increased or decreased graft survival when compared with the other ABO blood types $(P>.05)$. When looking at posttransplant graft survival in blood type B recipients (Figure 3), blood type $\mathrm{O}$ donor hearts were associated with decreased graft survival when compared with type B grafts $(P<.05)$.

\section{DISCUSSION}

Since the advent of cardiac transplantation in the 1960s, physicians have made considerable efforts to improve shortand long-term transplant outcomes by investigating the causes of graft rejection and generalized graft failure. Immunologically, as with other transplanted organs, this has involved minimizing antigenic mismatches between graft donors and recipients. Because of the high demand and comparatively low supply of available organs for transplant, emphasis has also been placed on generating graft allocation policies that are fair and effective. Because of the multifactorial nature of graft failure, these efforts have led to a debate about the impact of ABO blood type compatibility and the importance of HLA matching on adult heart transplant outcomes.

For HLA matching, Opelz and Wujciak ${ }^{10}$ definitively showed a strong relationship between donor-recipient 
TABLE 6. Multivariate Cox proportional hazards regression model

\begin{tabular}{|c|c|c|}
\hline Variable & $\begin{array}{c}\text { Hazard ratio }(95 \% \\
\text { confidence limits) }\end{array}$ & $P$ value* \\
\hline $\mathrm{ABO}$ compatible $\dagger$ & $0.99(0.89-1.10)$ & .87 \\
\hline Sex (male vs female) & $0.95(0.88-1.03)$ & .24 \\
\hline \multicolumn{3}{|l|}{ Donor ethnicitył } \\
\hline Black & $1.08(0.98-1.20)$ & .14 \\
\hline Hispanic & $1.00(0.90-1.10)$ & .95 \\
\hline Asian & $1.13(0.87-1.47)$ & .37 \\
\hline \multicolumn{3}{|l|}{ Recipient ethnicity $\ddagger$} \\
\hline Black & $1.42(1.30-1.56)$ & $<.001$ \\
\hline Hispanic & $1.09(0.94-1.25)$ & .25 \\
\hline Asian & $0.92(0.70-1.19)$ & .52 \\
\hline \multicolumn{3}{|l|}{ Life support at transplant $\S$} \\
\hline All & $1.07(0.94-1.21)$ & .32 \\
\hline IABP & $0.98(0.81-1.20)$ & .85 \\
\hline IV inotropes & $0.95(0.85-1.06)$ & .34 \\
\hline Ventilatory support & $1.88(1.50-2.37)$ & $<.001$ \\
\hline ECMO & $2.60(1.72-3.83)$ & $<.001$ \\
\hline Ischemic time & $1.09(1.06-1.13)$ & $<.001$ \\
\hline \multicolumn{3}{|c|}{ Wait list status at transplant $\|$} \\
\hline 1B & $1.00(0.90-1.11)$ & .95 \\
\hline 2 & $1.08(0.94-1.23)$ & .29 \\
\hline \multicolumn{3}{|l|}{ Status before transplant 9} \\
\hline In ICU & $1.24(1.10-1.39)$ & $<.001$ \\
\hline In hospital (not ICU) & $1.13(0.99-1.28)$ & .07 \\
\hline Total bilirubin & $1.03(1.02-1.04)$ & $<.001$ \\
\hline
\end{tabular}

HLA-A, HLA-B, and HLA-DR mismatches on posttransplant graft survival through the collaborative transplant study. More recent studies have demonstrated that the presence of circulating HLA-directed donor-specific alloantibodies are correlated with increased morbidity and mortality, cardiac allograft vasculopathy, and increased rates of graft rejection. ${ }^{11,12}$

Regarding $\mathrm{ABO}$ blood type matching, initial reports suggested that ABO-compatible transplants are less efficacious than ABO-identical ones. ${ }^{5,6}$ More recently, investigators have disagreed with this conclusion. ${ }^{7,8,13}$ A common problem of past studies has been a relatively small sample size precluding strong statistical power. In our analysis, we demonstrated that $\mathrm{ABO}$-identical and $\mathrm{ABO}$-compatible heart transplants have similar outcomes in terms of graft survival. By analyzing all adult cardiac transplants performed between 2000 and 2010, we were able to use a modern cohort of patients with a significantly larger sample size. Although our univariate analysis did show statistically significant differences in survival at 30 days and 1,3 , and 5 years posttransplant between the 2 study cohorts, these differences did not hold up after controlling for potential confounding variables in the multivariable models.
TABLE 7. Multivariable logistic regression model: 30-day graft failure

\begin{tabular}{|c|c|c|}
\hline Variable & $\begin{array}{c}\text { Odds ratio } \\
(95 \% \text { confidence limits })\end{array}$ & $P$ value* \\
\hline ABO compatible & $1.23(0.97-1.56)$ & .08 \\
\hline \multicolumn{3}{|l|}{ Life support at transplant $\dagger$} \\
\hline All & $1.89(1.40-2.54)$ & $<.001$ \\
\hline IV inotropes & $0.58(0.46-0.74)$ & $<.001$ \\
\hline Ventilatory support & $2.78(1.87-4.14)$ & $<.001$ \\
\hline ECMO & $7.53(4.18-13.55)$ & $<.001$ \\
\hline Ischemic time & $1.24(1.15-1.34)$ & $<.001$ \\
\hline \multicolumn{3}{|c|}{ Wait list status at transplant $\ddagger$} \\
\hline $1 \mathrm{~B}$ & $1.23(0.97-1.56)$ & .09 \\
\hline 2 & $1.68(1.19-2.36)$ & .003 \\
\hline \multicolumn{3}{|l|}{ Status before transplant $\S$} \\
\hline In ICU & $1.60(1.23-2.07)$ & $<.001$ \\
\hline In hospital (not ICU) & $1.16(0.86-1.56)$ & .34 \\
\hline Total bilirubin & $1.08(1.05-1.10)$ & $<.001$ \\
\hline
\end{tabular}

One of these possible confounding variables was Organ Procurement and Transplantation Network wait list status at transplant. Interestingly, according to our analysis, ABO-compatible heart recipients were more often status $1 \mathrm{~A}$ at transplant when compared with $\mathrm{ABO}$-identical recipients $(50.3 \%$ vs $37.8 \%)$. In addition, ABO-compatible recipients were more likely to be in the ICU and receiving several different mechanisms of life support, including ECMO, intra-aortic balloon pump, parenteral inotropes, and ventilator support, than $\mathrm{ABO}$-identical heart recipients. These data suggest that $\mathrm{ABO}$-compatible recipients are generally sicker than ABO-identical recipients, contributing to a worse prognosis. This is further supported by

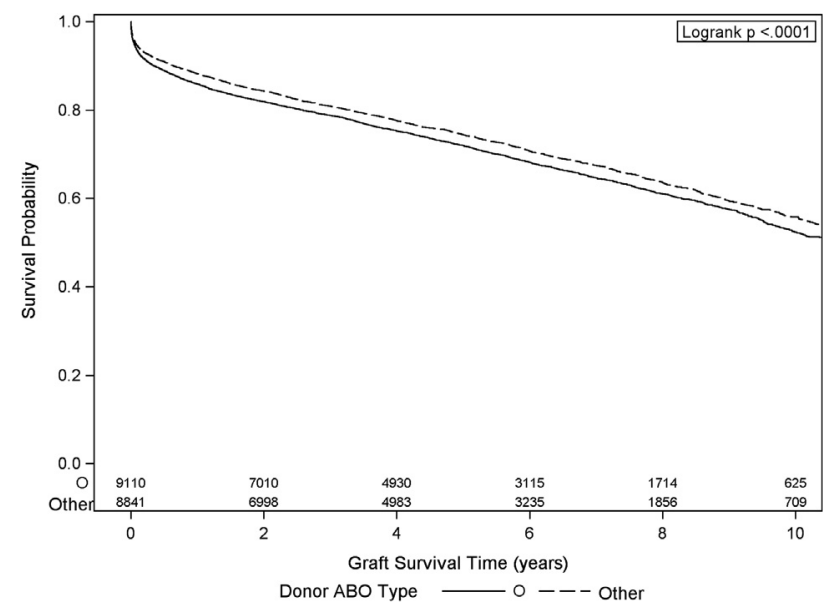

FIGURE 2. Kaplan-Meier graft survival analysis, donor type $\mathrm{O}$ versus all other heart transplants. Solid line, donor ABO type O; dashed line, all other donor types. A table is given with the number of patients at risk at each time point. The $P$ value corresponds to Mantel-Cox log-rank test results. 


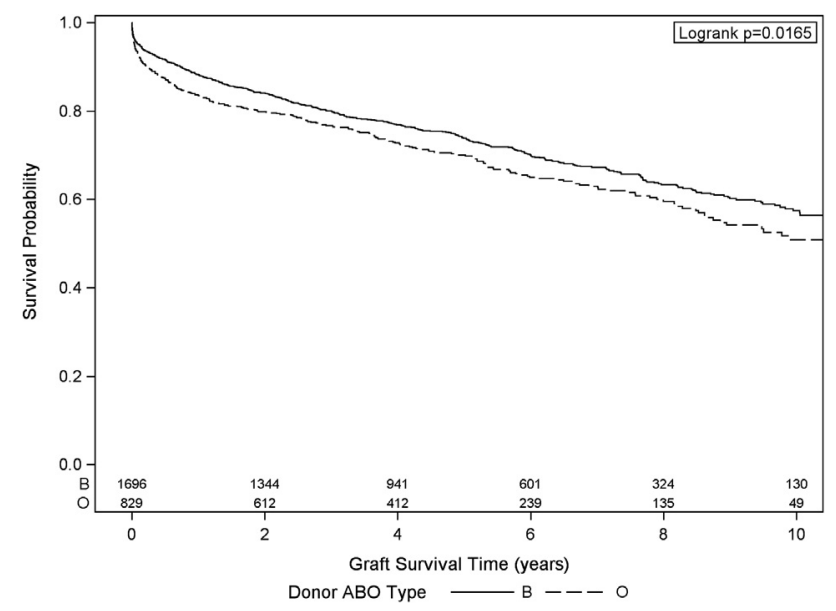

FIGURE 3. Kaplan-Meier graft survival analysis, donor type B versus donor type $\mathrm{O}$ heart transplants. Solid line, donor $\mathrm{ABO}$ type $\mathrm{B}$; dashed line, donor $\mathrm{ABO}$ type $\mathrm{O}$. A table is given with the number of patients at risk at each time point. The $P$ value corresponds to Mantel-Cox log-rank test results.

the fact that $\mathrm{ABO}-\mathrm{compatible} \mathrm{recipients} \mathrm{had} \mathrm{a} \mathrm{higher} \mathrm{pre-}$ transplant mean total bilirubin compared with ABOidentical recipients $(1.48$ vs $1.25 \mathrm{mg} / \mathrm{dL})$, indicating a greater degree of heart failure.

On analysis of recipient cause of death by $\mathrm{ABO}$ blood type matching, ABO-compatible recipients died as a result of primary graft failure more frequently than recipients of ABO-identical hearts $(8.7 \%$ vs $5.8 \%)$. Heart transplant recipient mortality due to primary graft failure is frequently associated with "marginal" donors or recipients. ${ }^{14}$ This seems to suggest that ABO-compatible transplants involve more marginal recipients and/or donors than ABOidentical transplants.

In multivariate analysis, $\mathrm{ABO}$ blood type matching (identical vs compatible) was not a statistically significant predictor of decreased cumulative or 30-day graft survival. Instead, the Cox proportional hazards model indicated recipient ethnicity (specifically, African American), life support at transplant (ventilator support and ECMO), graft ischemic time, total bilirubin, and recipient status before transplant to be significant predictors of decreased graft survival after transplant. The multivariate logistic regression model indicated many of these variables (ie, life support at transplant, ischemic time, bilirubin, and status before transplant) and wait list status as statistically significant predictors of graft failure within 30 days of transplant. Other studies have demonstrated similar results regarding risk factors for decreased survival and increased graft failure after heart transplants. ${ }^{15-17}$

The observed discrepancies in the effect of ABO compatibility on cardiac transplant outcomes between our univariate and multivariate models can be explained by investigating the impact of individual donor ABO blood types on graft survival. We discovered, in both our univariate and multivariate analyses, that donor $\mathrm{ABO}$ blood type $\mathrm{O}$ is associated with decreased graft survival when compared with all other types. Because blood type $\mathrm{O}$ donor grafts are transplanted into recipients of all blood types (Table 1), the poorer outcomes associated with type $\mathrm{O}$ donor hearts could be skewing the results of our univariate analysis to misleadingly suggest that ABO-compatible transplants result in worse outcomes than ABO-identical ones. We confirmed this hypothesis by removing all type $\mathrm{O}$ donors from our univariate analysis, which demonstrated no statistically significant difference in graft survival at all time points after transplant between ABO-identical and ABO-compatible cohorts $(P>.05)$.

The poor outcomes associated with type $\mathrm{O}$ donor grafts do have implications for organ allocation policies. According to the Organ Procurement and Transplantation Network policy 3.7.8.1 from February 2013, "Blood type O donors shall only be allocated to blood type $\mathrm{O}$ or blood type $\mathrm{B}$ patients" before being offered to blood type A or $\mathrm{AB}$ patients. Given the poor outcomes associated with blood type $\mathrm{O}$ grafts in type $\mathrm{B}$ recipients, this policy may need to be reviewed. Interestingly, previous studies have shown that blood type $\mathrm{O}$ individuals experience decreased rates of morbidity regarding conditions such as congestive heart failure. ${ }^{18}$ Further research should be conducted to investigate possible explanations for the poor outcomes associated with blood type $\mathrm{O}$ donor hearts and the best organ allocation scheme for managing these grafts.

\section{Limitations}

Such as any other retrospective cohort study, this investigation was limited by the strength of the primary database in terms of completeness, accuracy, quality, and appropriateness of the predictor variables. Although the data set provided by UNOS was extremely comprehensive and included many important variables that described baseline donor and recipient information and postoperative outcomes, the study could have been strengthened if additional data were available to us. Furthermore, because it is a large national database compiled over many years, the accuracy of all the patient information coded in the UNOS database cannot be guaranteed. We are confident, however, that given the nature of our investigation, an analysis of a large national cohort of patients, any errors in patient data will not bias our results.

\section{CONCLUSIONS}

In the past decade, $\mathrm{ABO}$-compatible donor hearts were preferentially given to sicker transplant recipients. As demonstrated in this study, transplantation using ABOcompatible adult hearts does not result in adverse outcomes with respect to graft survival and incidence of acute rejection compared with ABO-identical grafts. Therefore, ABO-compatible and ABO-identical heart transplant matches should be viewed equally in clinical decision 
making and to maximize efficiency within the available donor pool. This will help optimize the use of donor organs, an extremely important, yet scarce, resource. In doing so, waiting times could be shortened and overall outcomes could be improved. In addition, because ABO blood type $\mathrm{O}$ donor grafts are associated with decreased survival after transplant, current organ allocation policies should be reviewed, particularly those pertaining to $\mathrm{ABO}$ blood type $\mathrm{B}$ heart transplant recipients.

We thank Ms. Elsa Su, MS (Statistics), for helping in the preparation of the manuscript and the Yale School of Medicine Office of Student Research for support.

\section{References}

1. The International Society for Heart and Lung Transplantation. ISHLT transplant registry quarterly reports for heart in North America: survival rates for transplants performed between April 1, 2008, and March 31, 2012. Dallas, TX: ISHLT; 2012

2. Williams JA, Bethea BT. Heart transplantation and treatment of end-state heart disease. In: Conte JV, Baumgartner WA, Owens SG, Dorman T, eds. The Johns Hopkins Manual of Cardiac Surgical Care. 2nd ed. Philadelphia, PA: Mosby/ Elsevier; 2008:173-93.

3. Kirklin JK, Young JB, McGiffin DC. The donor heart. In: Heart Transplantation. Philadelphia, PA: Churchill Livingstone; 2002:293-339.

4. Subramanian V, Ramachandran S, Klein C, Wellen JR, Shenoy S, Chapman WC, et al. ABO-incompatible organ transplantation. Int J Immunogenet. 2012;39: 282-90.

5. McKenzie FN, Tadros N, Stiller C, Keown P, Sinclair N, Kostuk W. Influence of donor-recipient lymphocyte crossmatch and $\mathrm{ABO}$ status on rejection risk in cardiac transplantation. Transplant Proc. 1987;19:3439-41.

6. Nakatani T, Aida H, Frazier OH, Macris MP. Effect of ABO blood type on survival of heart transplant patients treated with cyclosporine. J Heart Transplant. 1989;8:27-33.

7. Kocher AA, Schlechta B, Ehrlich M, Ploner M, Khazen C, Schuster MP, et al. Effect of $\mathrm{ABO}$ blood type matching in cardiac transplant recipients. Transplant Proc. 2001;33:2752-4.

8. Neves C, Prieto D, Sola E, Antunes MJ. Heart transplantation from donors of different ABO blood type. Transplant Proc. 2009;41:938-40.

9. Stehlik J, Edwards LB, Kucheryavaya AY, Benden C, Christie JD, Dipchand AI, et al. The registry of the international society for heart and lung transplantation: 29th official adult heart transplant report-2012. J Heart Lung Transplant. 2012; 31:1052-64.

10. Opelz G, Wujciak T. The influence of HLA compatibility on graft survival after heart transplantation: the collaborative transplant study. N Engl J Med. 1994;330: 816-9.

11. Kaczmarek I, Deutsch MA, Kauke T, Beiras-Fernandez A, Schmoeckel M, Vicol C, et al. Donor-specific HLA alloantibodies: long-term impact on cardiac allograft vasculopathy and mortality after heart transplant. Exp Clin Transplant. 2008;6:229-35.

12. Stastny P, Lavingia B, Fixler DE, Yancy CW, Ring WS. Antibodies against donor human leukocyte antigens and the outcome of cardiac allografts in adults and children. Transplantation. 2007;84:738-45.

13. Sjogren J, Ljungdahl-Waller F, Senneby E, Ekmehag B, Koul B, Nilsson J. Heart transplantation with ABO-identical versus ABO-compatible cardiac grafts: influence on long-term survival. Scand Cardiovasc J. 2010;44:373-9.

14. Young JB, Naftel DC, Bourge RC, Kirklin JK, Clemson BS, Porter CB, et al. Cardiac Transplant Research Database Group. Matching the heart donor and heart transplant recipient: clues for successful expansion of the donor pool: a multivariable, multiinstitutional report. J Heart Lung Transplant. 1994;13:353-64; discussion 364-5.

15. Singh TP, Almond CS, Semigran MJ, Piercey G, Gauvreau K. Risk prediction for early in-hospital mortality following heart transplantation in the United States. Circ Heart Fail. 2012;5:259-66.

16. Allen JG, Weiss ES, Arnaoutakis GJ, Russell SD, Baumgartner WA, Conte JV, et al. The impact of race on survival after heart transplantation: an analysis of more than 20,000 patients. Ann Thorac Surg. 2010;89:1956-63; discussion 1963-4.
17. Kilic A, Weiss ES, George TJ, Arnaoutakis GJ, Yuh DD, Shah AS, et al. What predicts long-term survival after heart transplantation? an analysis of 9,400 ten-year survivors. Ann Thorac Surg. 2012;93:699-704.

18. He M, Wolpin B, Qi L, Manson JE, Rimm E, Hu FB, et al. ABO blood group and risk of coronary heart disease in two prospective cohort studies. Arterioscler Thromb Vasc Biol. 2012;32:2314-20.

\section{Discussion}

Dr Nader Moazami (Cleveland, Ohio). Well, let me start by congratulating you on your presentation. Many of us who were at your stage in our careers have not had the opportunity to present in such a prestigious forum, and I am hopeful that this bodes well for your future in academic surgery. I will start with a few general comments and then at the end ask my questions.

I think the topic and title of this talk are interesting. For many transplant clinicians, the issue of ABO-compatible transplantation seems to have been put to rest, particularly in the era of continued organ shortage. In fact, the current trend in the literature is largely focused on a completely opposite and different strategy, that is, ABO-incompatible transplantation. This strategy has been widely applied in the renal world and has led to an increase in the number of living-related kidney transplantations. A similar strategy has been successfully applied in the neonatal and pediatric heart transplant population for whom the availability of organs in a timely fashion is limited.

Now, most of us think of $\mathrm{ABO}$ as antigens expressed on the surface of red blood cells, but in fact they are widely expressed on endothelial cells, including those of the heart. The specter of antibody-mediated hyperacute rejection is what has made ABOincompatible heart transplantation a hurdle in adults and, in the current era of LVADs, of much less interest. So the question is, where does ABO-compatible heart transplantation fit in our overall practice and how important is this in terms of graft and patient survival, a question that you have attempted to analyze in your presentation today.

I am going to draw 3 broad conclusions from your presentation and follow those with questions, and I will wait for your answer for each one.

As you know, graft failure is multifactorial and depends on many donor and recipient variables, some of which you have accounted for in your analysis, particularly those markers that identify sicker patients. I am not sure if in your analysis other well-known variables, such as pulmonary hypertension, recipient and donor age, and ischemic times, have been accounted for. Did you look at any of these factors for graft failure in your multivariable model?

Mr Jawitz. First of all, thank you, Dr Moazami, for your enthusiastic support and criticism. As a medical student planning to pursue a career in surgery, it really means a lot to me.

To answer your question, we were able to look at some of those variables in the database. Unfortunately, we were limited by the variables that were coded in the UNOS database, and in some instances there was a significant amount of data missing. In our multivariate analysis, however, we actually did show that total bilirubin time, ethnicity, and a number of other variables were actually independently associated with poorer outcomes. Pulmonary hypertension was included in our cause of death analysis, but not in our pretransplant univariate analysis of recipient baseline characteristics. 
Dr Moazami. That brings me to the second conclusion that you drew, and that is that ABO-compatible heart transplantation is an acceptable strategy and unlikely to impact short- or long-term survival. In fact, the International Society of Heart and Lung Transplantation, which is a large registry of all transplant recipients, on an annual basis evaluates all transplant-related data. Interestingly, occasionally a small improved survival difference has been seen between ABO-identical and ABO-compatible donors. However, this generally has been negligible and largely accounted for by many other markers of immunogenicity, namely, the degree of HLA mismatching. This brings me to the next question for you.

In looking at short- and long-term results, the immunogenicity of MHC antigens and also presence of donor-specific antibodies play a large role in graft viability. In your analysis, were any of these factors, specifically HLA matching, panel-reactive antibody levels, or cross match results, available and accounted for?

Mr Jawitz. Thank you very much. That is an excellent question. Yes, it is true that HLA matching is extremely important in long-term graft outcomes. I believe, in the last 10 years, research conducted by Opelz and colleagues, the collaborative transplant study, showed that 2 HLA-A, HLA-B, or HLA-DR mismatches were actually associated with $25 \%$ increased graft failure compared to 0 or 1 mismatch within 3 years of transplant. In terms of our study, we really wanted to keep it simple and specifically focus on $\mathrm{ABO}$ blood types, namely, ABO-compatible versus $\mathrm{ABO}-$ identical matches. In addition, a recently published article specifically looking at renal transplantation showed no correlation between HLA matching and ABO blood type matching.

I do agree that HLA type would be interesting to look at and see, specifically, how differences in HLA blood type between donors and recipients have impacted these data.

Dr Moazami. Finally, I caution against one of your conclusions, which is regarding decreased survival of blood type $\mathrm{O}$ donors for, specifically, blood type $\mathrm{B}$ recipients. The UNOS organization mandate is based on 2 major premises: (1) the equity in organ allocation and (2) in maximizing the survival benefit for recipients that are at the highest risk of dying. The policy of allocating $\mathrm{O}$ donor hearts to type $\mathrm{O}$ recipients first and then type $\mathrm{B}$ is a reflection of this policy. Type $\mathrm{O}$ recipients can only receive organs from type $\mathrm{O}$ and, hence, typically have longer wait times on the list. Similarly, type B in the United States comprises only about $10 \%$ of the population and, hence, they are at a disadvantage compared to type A blood that comprises about $40 \%$ of the population.

So, with the Kaplan-Meier curve that you showed at the end in terms of the blood donor $\mathrm{O}$ to $\mathrm{B}$ recipients, were any of these risk adjusted, and is it possible that if we correct for some of the factors that I mentioned previously related to immunogenicity that these small differences in survival will disappear?

Mr Jawitz. Yes, that is a good question. We actually looked at primary graft failure as an outcome between donor type $\mathrm{O}$ hearts going to type B individuals and type B hearts going to type B recipients, and we actually found out that compatible matches, that is, type $\mathrm{O}$ hearts going to type $\mathrm{B}$ recipients, were actually associated with increased rates of primary graft failure.

As for the current OPTN policy and organ allocation scheme, you are right, the reason that blood type $B$ recipients preferentially receive type $\mathrm{O}$ organs is because of the short supply of type $\mathrm{B}$ hearts in this country. When approaching a potential change in policy, it would be important to ensure that that any decrease in donor heart availability for type B recipients would be more than made up for by significantly improved posttransplant outcomes. More analysis is certainly needed before we would feel comfortable recommending such a policy.

Dr Moazami. Thank you.

Dr David McGiffin (Birmingham, Ala). I just want to follow on from that point. You mentioned in your manuscript and in your talk about the poorer survival of blood $\mathrm{O}$ to $\mathrm{B}$. You have demonstrated that there are no immunological consequences of that, but $\mathrm{O}$ to $\mathrm{B}$ is most likely a surrogate for sicker donors and sicker recipients. On that basis, though, how would you change the allocation system for what is probably an immutable problem?

Mr Jawitz. That is a good question. As Dr Moazami pointed out, the reason why blood type $\mathrm{B}$ recipients are receiving blood type $\mathrm{O}$ donor hearts is because of the comparative shortage of type $\mathrm{B}$ donor hearts in this country. To really change the current allocation scheme, we would have to take into account the potential of actually harming type B individuals by changing the policy. I do not think there is a really great answer to how to specifically change the policy at this point and I certainly believe that more research is warranted.

The senior author on this paper actually believes that changing the pretransplant management of type B patients, that is instead of rushing to transplant them with a type $\mathrm{O}$ donor heart, perhaps using an LVAD as a bridge to transplant as you wait for an identical type B donor graft, may be a method of mitigating this problem.

Dr Pieter Kappetein (Rotterdam, The Netherlands). You showed that there is a significant difference between the different groups, especially for the blood type $\mathrm{O}$ patients. Of course, this is a large group of patients. For example, in the A-B group, you have few patients, and might that not be a type II error that maybe there might also be a difference there because you do not have enough patients that you do not see the difference?

Mr Jawitz. You are absolutely right. We were limited by the database that we had and in several instances due to incomplete data and a lack of certain variables, we were unable to answer all of our questions.

Dr Kappetein. And, of course, therefore, in a group where you have enough patients you can identify easier a difference and while in the groups that are smaller there might be a difference as well but it might be more difficult to identify.

Mr Jawitz. Yes, you are correct. 